Ferroelectric characterization of aligned barium titanate nanofibres

This article has been downloaded from IOPscience. Please scroll down to see the full text article.

2013 J. Phys. D: Appl. Phys. 46105304

(http://iopscience.iop.org/0022-3727/46/10/105304)

View the table of contents for this issue, or go to the journal homepage for more

Download details:

IP Address: 193.137.16.115

The article was downloaded on 30/04/2013 at 15:51

Please note that terms and conditions apply. 


\title{
Ferroelectric characterization of aligned barium titanate nanofibres
}

\author{
Pedro Sá ${ }^{1}$, Jose Barbosa ${ }^{1}$, Igor Bdikin ${ }^{2}$, Bernardo Almeida ${ }^{1}$, \\ Anabela G Rolo ${ }^{1}$, Etelvina de Matos Gomes ${ }^{1}$, Michael Belsley ${ }^{1}$, \\ Andrei L Kholkin ${ }^{3}$ and Dmitry Isakov ${ }^{1}$ \\ ${ }^{1}$ Center of Physics, University of Minho, Campus de Gualtar, 4710-057 Braga, Portugal \\ ${ }^{2}$ Department of Mechanical Engineering and TEMA, University of Aveiro, 3810-193 Aveiro, Portugal \\ ${ }^{3}$ Department of Materials and Ceramics Engineering and CICECO, University of Aveiro, 3810-193 \\ Aveiro, Portugal \\ E-mail: dmitry@ fisica.uminho.pt
}

Received 30 July 2012, in final form 4 January 2013

Published 12 February 2013

Online at stacks.iop.org/JPhysD/46/105304

\begin{abstract}
We report the synthesis, structural and ferroelectric characterization of continuous well-aligned nanofibres of barium titanate produced by the electrospinning technique. The fibres with average diameter of 150-400 nm consist of connected nanoparticles of $\mathrm{BaTiO}_{3}$ stacked together to form the shape of a long filament. The tetragonal phase in the obtained nanofibres was revealed by the x-ray diffraction and Raman spectroscopy and has been also confirmed by the second harmonic generation (SHG) and piezoresponse force microscopy (PFM). The temperature dependence of the SHG in the vicinity of the paraelectric-ferroelectric phase transition suggests that barium titanate nanofibres are indeed ferroelectric with an apparent glass-like state caused by metastable polar nanoregions. The existence of domain structure and local switching studied by PFM present clear evidence of the polar phase at room temperature.
\end{abstract}

(Some figures may appear in colour only in the online journal)

\section{Introduction}

Nanostructured barium titanate $\left(\mathrm{BaTiO}_{3}\right)$ in the form of dots, wires and rods is being actively developed for applications in multilayer capacitors and tunable microwave transducers $[1,2]$. The tetragonal phase, which is stable in the vicinity of room temperature $\left(10-120^{\circ} \mathrm{C}\right)$, makes $\mathrm{BaTiO}_{3}$ attractive for electromechanical conversion and ferroelectric random access memory cells. Recent developments in high-resolution microscopy and ultrafast pulsed lasers make barium titanate nanoparticles potential nonlinear optical nanoprobing elements for tissue bio-imaging [3,4]. Unlike two-photon fluorescence probes, noncentrosymmetric labels such as $\mathrm{BaTiO}_{3}$ nanoparticles are not subject to photobleaching. Furthermore, the second harmonic generated light has increased optical contrast wavelength since it is far removed from the excitation. Additionally, nanoparticles have a less stringent dependence on phase matching conditions [5].

Nanostrutured barium titanate can also be used as a humidity sensor [6], or gas and chemical compound sensing element with good reversibility and sensitivity
$[7,8]$. The sensing mechanism relies on changes of the dielectric permittivity (capacitive-type sensor) or conductivity (conductive-type sensor), due to the adsorption of the probed vapour organic compound, and dipole induced interfacial polarization potential. The conductivity of the $\mathrm{BaTiO}_{3}$-based sensors can vary from dielectric (nonconductive) to a highly conductive state resulting in excellent chemical selectivity and sensitivity. To increase the performance of the mentioned active nanoelements, a higher aspect ratio is preferable, since one-dimensional nanofibre structures with high surface-tovolume ratio can increase the electromechanical coupling in nanocomposites [9] and facilitate adsorption of molecules on the surface of the sensors [6].

In spite of the fact that several approaches and methods for the production of $\mathrm{BaTiO}_{3}$ nanowires and nanofibres have been recently implemented [10-13], a comprehensive study of the ferroelectric properties of these nanostructures is very limited $[14,15]$. However, the need for the integration of barium titanate with nanoscopic devices makes the development of reliable $\mathrm{BaTiO}_{3}$ nanostructures with tetragonal ferroelectric structure very important. Recently, in our group the 
preparation of barium titanate nanofibres was attempted [16] using a procedure similar to that described by Yuh et al [11] and later by Sahoo and Panda [17]. In the $\mathrm{BaTiO}_{3}$ nanofibres obtained by such a method, the investigation of the ferroelectric switching has been attempted; however, no saturation of the hysteresis loop was obtained. The work described in this communication is devoted to the improvement of the processing approach targeted to obtain well-aligned nanofiberlike structures of $\mathrm{BaTiO}_{3}$ with pronounced ferroelectric properties. Due to the small nanoscopic size of the particles composing the fibres, the tetragonal (ferroelectric) distortion of the barium titanate crystallites is reduced due to the presence of a non-ferroelectric grain boundary layer (dead layer) on the grain surface $[18,19]$. Therefore, fabrication of the nanofibres with well-aligned morphology and strong ferroelectric responses is technologically challenging.

Additionally, the production of well-aligned nanofibre array structure is presented. In contrarst to the conventional electrospinning process, where the nanofibres are collected in a random mesh, the aligned bundles of nanofibres are more favourable for applications of these complex oxides systems for integration with planar electronics, allowing an easier and more precise electrode deposition. Also, the aligned structure will allow increasing the efficiency of the bundles as compared with a random structure due to the improved packing and larger surface area.

\section{Experimental}

To prepare the electrospinning precursor solution, $2 \mathrm{~g}$ of poly(vinyl pyrrolidone) (PVP, $\left.M_{\mathrm{w}}=43000\right)$ was dissolved in $3 \mathrm{ml}$ of ethanol. $1.15 \mathrm{~g}$ of titanium iso-propoxide ( Ti $\left[\mathrm{OCH}\left(\mathrm{CH}_{3}\right)_{2}\right]_{4}$ ) was added to $3 \mathrm{ml}$ acetic acid, where $1 \mathrm{~g}$ of barium acetate $\left(\left(\mathrm{CH}_{3} \mathrm{COO}\right)_{2} \mathrm{Ba}\right)$ was previously dissolved. Subsequently, the acidic solution was mixed with the PVPalcohol solution and the obtained mixture was stirred for several hours prior electrospinning. During electrospinning the applied electric tension was $15 \mathrm{kV}$, with $10-12 \mathrm{~cm}$ distance between syringe needle and collector and $1 \mathrm{ml} \mathrm{h}^{-1}$ of the flow rate. To obtain aligned $\mathrm{BaTiO}_{3}$ nanofibres, a grounded rotating drum (600-6000 r.p.m.) was used as a collector. The aselectrospun fibres then were dried at $100^{\circ} \mathrm{C}$ for $1 \mathrm{~h}$ in order to vaporize the carrier polymer. The $\mathrm{BaTiO}_{3}$ grain size within the fibres can be affected by the annealing temperature [20]. In contrast to previous work [16], here, the annealing time and temperature were increased up to $12 \mathrm{~h}$ and to $900-1100^{\circ} \mathrm{C}$, respectively. Since the presence of the cubic $\mathrm{BaTiO}_{3}$ phase is favoured for smaller grain sizes, the changes introduced on the preparation routine aim to decrease the non-ferroelectric grain boundary layer on the $\mathrm{BaTiO}_{3}$ grains surface [20].

The morphology of the electrospun $\mathrm{BaTiO}_{3}$ fibres was observed under a scanning electron microscope (SEM, Nova NanoSEM 200) after gold coating. The structural studies were performed by $\mathrm{x}$-ray diffraction (XRD) with a Bruker D8 Discover powder diffractometer using $\mathrm{CuK} \alpha$ radiation $(40 \mathrm{kV}, 50 \mathrm{~mA})$. Raman measurements were performed in a backscattering configuration, using the $488 \mathrm{~nm}$ beam from an argon ion laser focused on the fibre mesh plane. The scattered
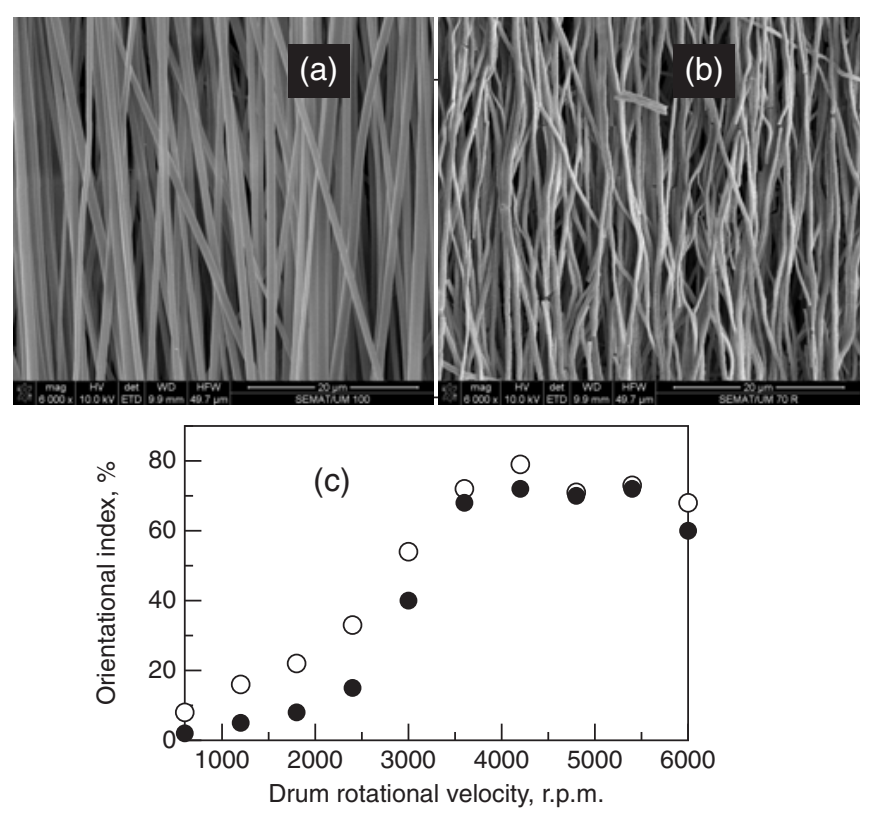

Figure 1. SEM images of the barium titanate fibres $(a)$ As-electrospun and $(b)$ after annealing; $(c)$ evolution of the alignment degree (orientational index) of the nanofibres with the rotation velocity of the collector drum. Open and filled circles correspond to as-electrospun and annealed nanofibres, respectively.

light was dispersed by a subtractive spectrometer and collected with a liquid-nitrogen-cooled charge coupled device (CCD) detector.

The second harmonic generation measurements were carried out on $\mathrm{BaTiO}_{3}$ nanofibre mat using a Q-switched Nd:YAG laser (LPY604T-10, Litron) with $7 \mathrm{~ns}$ pulse length and $10 \mathrm{~Hz}$ repetition rate. The experiment was performed in a transmission mode on fibers preliminarily deposited on a supporting optical glass mounted on the controllable heating cell. The linearly polarized focused beam, with $\lambda=1064 \mathrm{~nm}$ and pulse power $\sim 3 \mathrm{MW}$, was incident normally to the plane of the fibre mat. Appropriate colour and interference filters were employed to discriminate against the scattered infrared fundamental light and SHG emission was detected by a photomultiplier connected to a gated Boxcar integrator.

The ferroelectric properties and domain structure of the $\mathrm{BaTiO}_{3}$ nanofibres were studied using piezoelectric force microscopy. Prior to the measurements, the barium titanate nanofibres were deposited and aged on a conductive platinum substrate. The piezoelectric response was implemented by atomic force microscope (Multimode, Bruker) equipped with a lock-in amplifier (SR830, Stanford Research). The electric voltage was applied with a conductive cantilever (Nanosensors) by means of function generator (FG120, Yokogawa) and a voltage amplifier (7602, Krohn-Hite).

\section{Results and discussion}

\subsection{Morphological and structural studies}

The alignment bundles of electrospun nanofibres have been produced using a rotating collector drum [21]. Figures 1(a)(b) show aligned nanofibre arrays for the as-electrospun 

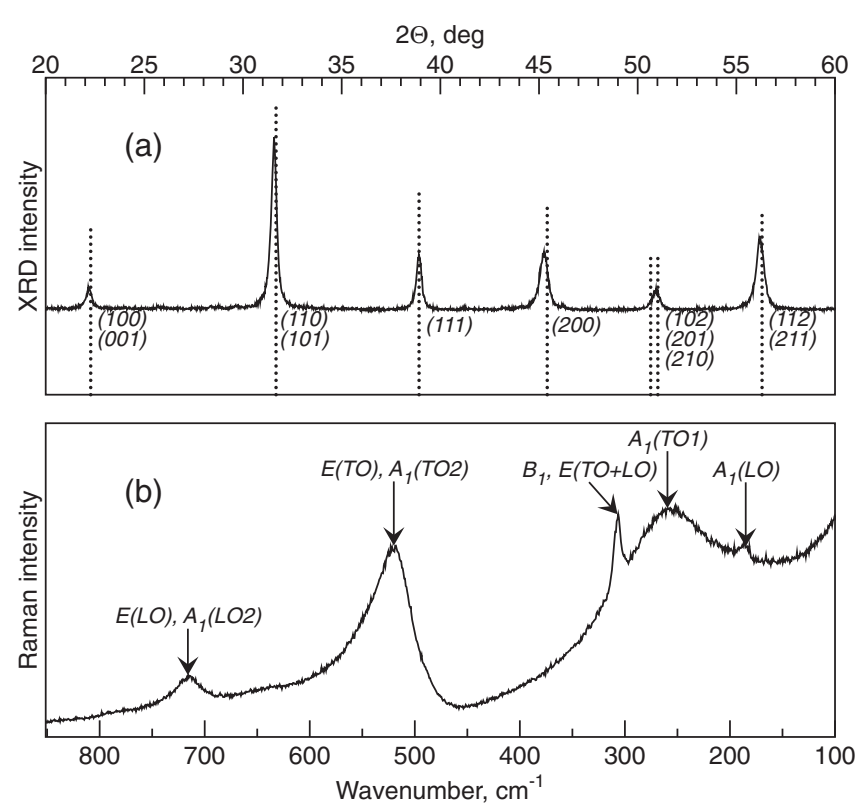

Figure 2. (a) XRD pattern measured on the annealed barium titanate nanofibre mat; $(b)$ corresponding Raman spectrum.

and annealed samples, respectively. The as-electrospun nanofibres present a smooth polymer surface with lengths up to centimeters and diameters in the range $200-600 \mathrm{~nm}$. To obtain the $\mathrm{BaTiO}_{3}$ tetragonal phase the fibres were then calcined at $900{ }^{\circ} \mathrm{C}$ during $12 \mathrm{~h}$ resulting in the multi-grain conglomerate that formed a continuous fiber-like structure with the final fibre diameter reduced to $150-400 \mathrm{~nm}$ due to the heat treatment (figure 1(b)).

The study of the degree of fibre alignment was made on the fibre mats before and after the annealing treatment. The quantitative evaluation of the alignment degree was carried out by a $2 \mathrm{D}$ discrete Fourier transform image analysis technique (see details in [22,23]). In this method the SEM image of the nanofibres is transformed into a complex image in which the high frequencies are grouped at the centre, while the low frequencies are located at the edges. The information in this 2D DFT image was mapped into plots of the spatial frequency averaged intensity as a function of the angle. From such plots the orientational index OI of each image is calculated, which characterizes the fraction of parallel fibres (OI $=100 \%$ corresponds to totally parallel aligned fibres and $0 \%$ for a completely random orientation of the fibers).

Figure 1(c) shows the evolution of the orientational index with the rotation velocity of the collector drum before and after annealing. For a low velocity of rotation the fibres are disoriented, with no prominent fibre orientation. As the rotation speed increases, the fibre alignment of both asprepared and annealed fibres increases significantly so that the OI attains a value as high as $76 \%$ when the rotation velocity is $3600 \mathrm{rpm}$. At speeds above $3600 \mathrm{rpm}$, the fibre orientation remains relatively strong, slightly decreasing at $6000 \mathrm{rpm}$.

The XRD spectra (figure 2) obtained in annealed fibres presents well-defined diffraction peaks characteristic of the perovskite structure of a polycrystalline barium titanate. The XRD peaks were fitted with Voight functions in order to determine peak positions and widths. The grain size of the barium titanate fibres was calculated from the (1 111$)$ XRD peak width, using the Scherrer equation [24]. The determined average size of the $\mathrm{BaTiO}_{3}$ grains composing the fibres was $58 \mathrm{~nm}$, as estimated from SEM micrographs. These grains are above the critical size $(\sim 30 \mathrm{~nm})$ below which the stabilization of the tetragonal phase at room temperature is hindered [19].

Due to the small size of the particles composing the fibres the diffraction peaks are broad and it is difficult, from the XRD results alone, to distinguish which of the $\mathrm{BaTiO}_{3}$ phases (cubic or tetragonal) is dominant. In fact, it is known that for grain sizes below $1 \mu \mathrm{m}$ the tetragonal distortion of the barium titanate crystallites is reduced due to the presence of a non-ferroelectric grain boundary layer on the grains surface [25-27]. However, we observe a broadening of the diffraction (1 10$)$ and (200) peaks, as compared for example with the (1 11 1) peak, along with an increasing asymmetry of these peaks towards the higher angles suggesting the stabilization of the polar phase of $\mathrm{BaTiO}_{3}$.

To confirm the presence of this polar phase in the annealed barium titanate nanofibre mats and to further characterize their structure, Raman spectroscopy measurements have been performed. Raman spectroscopy yields phonon parameters reflecting the local crystal symmetry, allowing the detection of the presence of the structure distortion of the grains unit cell. Figure 2 presents the measured Raman spectrum obtained on the $\mathrm{BaTiO}_{3}$ nanofibres. The cubic (paraelectric) phase of $\mathrm{BaTiO}_{3}$ has a $\mathrm{Pm} 3 m$ crystal symmetry, which, theoretically does not have Raman active modes [28, 29]. However, distortions to the perfect cubic cell induced by disorder at the $\mathrm{Ti}$ positions or due to external stresses over the grains can occur and broad peaks at around 250 and $520 \mathrm{~cm}^{-1}$ have been observed on polycrystalline powders [30]. The tetragonal form of $\mathrm{BaTiO}_{3}$ belongs to the $P 4 \mathrm{~mm}$ space group with corresponding optical modes transformed according to the irreducible representations $3\left(A_{1}+E\right)+E+B_{1}$ where modes $E$ are doubly degenerate and the modes $A_{1}$ and $B_{1}$ are nondegenerate giving twelve long wavelength optical modes. The bands at $185,272,307,520$ and $716 \mathrm{~cm}^{-1}$ are assigned to the $A_{1}(T O 1), B_{1}, E(T O+L O), E(T O), A_{1}(T O 2), E(L O)$ and $A_{1}(L O 2)$ vibrational modes, respectively, characteristic for the tetragonal symmetry of the $\mathrm{BaTiO}_{3}$ [30], which indicates the presence of a polar $\mathrm{BaTiO}_{3}$ phase in the fabricated samples.

\subsection{Ferroelectric behaviour}

The nonlinear optical phenomenon, such as optical SHG, is an additional tool for the characterization of the materials structure. SHG is only allowed in non-centrosymmetric media making it an important tool to characterize acentric phases. In SHG, the frequency doubled waves are generated with a nonlinear polarization that oscillates with the double of the excitation frequency $P_{i}^{2 \omega}=\chi_{i j k} E_{j}^{\omega} E_{k}^{\omega}$, where $\chi_{i j k}$ is the nonlinear susceptibility and $E_{j}^{\omega}$ and $E_{k}^{\omega}$ are the components of the incident radiation [31]. The SHG intensity reflects the behaviour on the averaged nonlinear polarization of local 


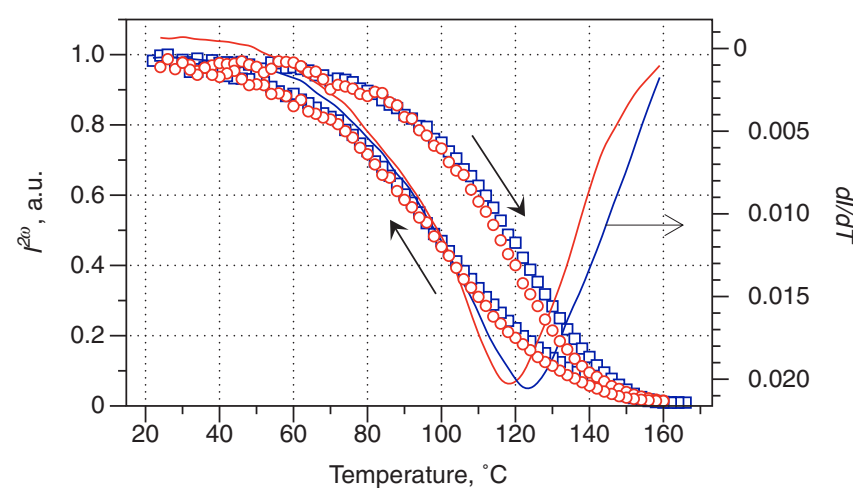

Figure 3. Normalized SHG intensity as a function of temperature observed in nanofibres of $\mathrm{BaTiO}_{3}$ calcined at $900{ }^{\circ} \mathrm{C}$ (squares) and $1100{ }^{\circ} \mathrm{C}$ (circles). The solid curves are the derivatives of $I(T)$.

regions in the illuminated volume $I^{2 \omega} \propto n^{2}\left|\sum \chi_{i j k} E_{j}^{\omega} E_{k}^{\omega}\right|^{2}=$ $\left\langle P^{2 \omega}\right\rangle^{2}$, where $n$ is the number of polar regions in the focal volume. In bulk ferroelectric materials where the spontaneous polarization $P_{\mathrm{s}}$ is the primary order parameter of the transition and the temperature dependence of the susceptibility is mainly due to the behaviour of $P_{\mathrm{s}}$, such as in barium titanate [31], the nonlinear polarizability is proportional to the spontaneous polarization [25,31-34]. Therefore, the temperature dependence of the SHG intensity $I^{2 \omega}(T)$ in these materials yields information on the temperature-dependent polarization $[33,35,36]$. Additionally, the technique also allows noncontact probing of ferroelectric properties when wiring is extremely difficult or impossible.

Here, prior to the SHG measurements, $\mathrm{BaTiO}_{3}$ nanofibres were transferred to the microscope glass slide. The bright green second harmonic emission from a thin $\mathrm{BaTiO}_{3}$ nanofibre mat was observed, indicating the presence of a noncentrosymmetric ferroelectric phase. The nonlinear optical effective susceptibility of $\mathrm{BaTiO}_{3}$ fibres was evaluated at room temperature by the powder Kurtz technique with the potassium dihydrogen phosphate as a reference sample. It is found to be about $12.5 \mathrm{pm} \mathrm{V}^{-1}$ and its value is comparable to those reported in the literature [37].

Figure 3 presents the temperature dependence (with heating/cooling rates of $1 \mathrm{~K} \mathrm{~h}^{-1}$ ) of the normalized SHG intensity $I^{2 \omega}(T)$ observed on the barium titanate nanofibres calcinated at $900^{\circ} \mathrm{C}$ and $1100^{\circ} \mathrm{C}$ during $12 \mathrm{~h}$. Increasing the annealing time from $1 \mathrm{~h}$ (as was done in [16]) to $12 \mathrm{~h}$ has allowed us to obtain $\mathrm{BaTiO}_{3}$ fibres with a clear ferroelectric response that can be detected in fibres annealed at temperatures above $800^{\circ} \mathrm{C}$. As seen from figure 3 , the $I^{2 \omega}(T)$ dependence is very broad and displays a diffuse ferroelectric-paraelectric phase transition behaviour. Such a broad transition with detectable SHG up to $900 \mathrm{~K}$ was observed in $\mathrm{BaTiO}_{3}$ in the form of ceramic and powder with an average size of $0.5 \mu \mathrm{m}$ $[34,38]$.

Since the intensity of the SHG follows mainly the behaviour of the spontaneous polarization, such observed gradual $I^{2 \omega}(T)$ decrease can be explained by the disordered distribution of the polar orientation in the $\mathrm{BaTiO}_{3}$ grains composing the nanofibres (assuming them to be metastable polar nanoregions with paraelectric interfaces
[39]). Additionally, we believe that a significant contribution to the SHG signal in paraelectric barium titanate is due to the large amount of the defects on the particle surface and possible coherence of the particle interfaces $[34,40]$. Such defects act like pinning off-centers and are related to the local remaining polar regions. This is also supported by the pronounced thermal hysteresis characteristic for relaxor ferroelectrics with nonequilibrium polar state. The possible contribution of the surface SHG (that originates from the local inversion symmetry breaking at the surface layer) in $\mathrm{BaTiO}_{3}$ nanofibres is rather weak and would not be expected to show the hysteresis type dependence on temperature that we observe close to the phase transition temperature. Since the surface cubic layer remains the same during the phase transition, any surface contribution to the second harmonic signal should be roughly constant over the range of temperatures explored in figure 3 . The strong variations in the SHG intensity are consistent with temperature-induced changes in the polarization of the tetragonal nanograins core, but are incompatible with a signal that has a large contribution from the local inversion symmetry breaking at the surface of the nanograins.

The Curie temperature was extracted from the minimum of the derivative of $I^{2 \omega}(T)$ dependence where most of the variation of the polarization occurs during the transition. The difference in $T_{\mathrm{c}}$ for the fibres annealed at different temperatures $\left(119{ }^{\circ} \mathrm{C}\right.$ and $123{ }^{\circ} \mathrm{C}$ for $\mathrm{BaTiO}_{3}$ fibres annealed at $900{ }^{\circ} \mathrm{C}$ and $1100^{\circ} \mathrm{C}$, correspondingly) can be understood in terms of the $T_{\mathrm{c}}$ dependence on the grain size. Increasing the annealing temperature in nano- and micrograined barium titanate apparently leads to increasing of their average grain size [16] with accompanying shift of the tetragonalcubic phase transition temperature to higher temperatures $[19,41,42]$.

Another effective tool for probing local ferroelectric properties of nanostructures is a piezoresponse force microscopy (PFM) [43,44]. In this technique, the conventional AFM is used to visualize the electromechanical response caused by an external ac electric field applied via a conducting tip. In contrast to far-field SHG, PFM allows local polarization imaging and characterization by means of local piezoresponse analysis.

The previous attempts to switch the polarization in the $\mathrm{BaTiO}_{3}$ fibers failed due to breakdown under the electric field [16]. A possible explanation for this failure is that defects induced by the growth process or ageing pin the polarization state such that it is then impossible to reverse. Increasing the annealing time (while maintaining the temperature $900-1000{ }^{\circ} \mathrm{C}$ ) from 1 to several hours results in fewer defects and a lower concentration of stresses at the interfaces causing the enhancement of the local field and subsequent breakdown. Here, the PFM image reveals a pronounced electromechanical response sensed by the AFM tip on the $\mathrm{BaTiO}_{3}$ nanofibre (figure 4) with prevalent polarization orientation normal to the substrate surface (via vertical PFM). The observed out-of-plane piezoresponse hysteresis loop shows full switching of the polarization with coercive bias about $\pm 5 \mathrm{~V}$. The quantitative measurement of the effective piezoelectric longitudinal coefficient value was 
(a)

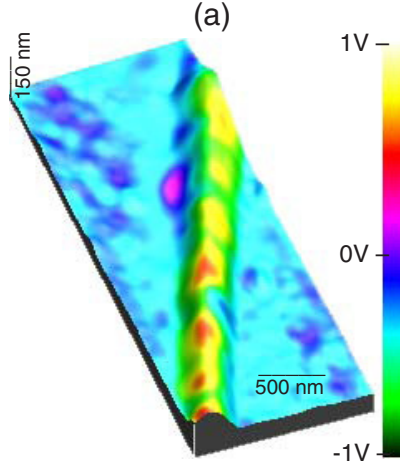

(b)

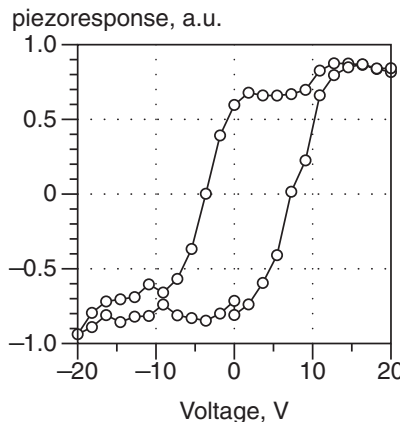

Figure 4. As-grown ferroelectric domains seen on the piezoresponse image $(a)$ and out-of-plane piezoresponse hysteresis loop $(b)$ of the $\mathrm{BaTiO}_{3}$ single nanofibre annealed at $900{ }^{\circ} \mathrm{C}$ during $12 \mathrm{~h}$.

more problematic because of the complex distribution of the applied electric field due to the cylindrical geometry of the $\mathrm{BaTiO}_{3}$ nanofibre and a coupling between vertical and lateral responses. The magnitude of the effective piezoelectric coefficient was evaluated by comparison with the PFM signal calibrated using a PZT thin film, giving a value about $20 \mathrm{pm} \mathrm{V}^{-1}$ for a fibre with $250 \mathrm{~nm}$ in diameter.

\section{Conclusion}

Continuous polycrystalline $\mathrm{BaTiO}_{3}$ nanofibres have been produced by the electrospinning technique from a sol-gel precursor solution and posterior calcination. The improvement of the processing routine, namely, increasing the calcination time and temperature makes it possible to obtain $\mathrm{BaTiO}_{3}$ nanofibres with pronounced ferroelectric behaviour. The use of a rotating drum as the electrospinning fibre collector allowed producing well-aligned $\mathrm{BaTiO}_{3}$ fibre bundles.

The presence of the tetragonal (ferroelectric) $\mathrm{BaTiO}_{3}$ phase was confirmed by Raman spectroscopy, second harmonic generation and piezoresponse force microscopy. The effective nonlinear optical coefficient of our barium titanate nanofibres has been found to be as strong as that for the conventional nonlinear optical bulk oxides. The temperature dependence of the second harmonic generation intensity $I^{2 \omega}(T)$ allowed the determination of the ferroelectricparaelectric transition temperature and confirmed polar behaviour of the $\mathrm{BaTiO}_{3}$ nanoparticles composing the nanofibres. PFM experiments provided clear evidence of the existence of polar phase at room temperature.

\section{Acknowledgments}

This work has been done in the framework of the CIÊNCIA-2007 programme (reference UMINHO-CF-06) and was financially supported by the European Regional Development Fund (ERDF) through Programa Operacional Factores de Competitividade (COMPETE: FCOMP-01-0124FEDER-014628), by the Portuguese Foundation for Science and Technology (FCT) (PTDC/CTM-NAN/114269/2009, PTDC/CTM-NAN/115125/2009, PTDC/CTM-CER/115085/

2009, PTDC/CTM/105597/2008, PTDC/CTM/099415/2008) and by the strategic project PEST-C/FIS/UI607/2011.

\section{References}

[1] Scott J F 1998 Ann. Rev. Mater. Sci. 2879

[2] Scott J F 2007 Science 315954

[3] Wang L, He Y, Hua J, Qi Q and Zhang T 2011 Sensors Actuators B 153460

[4] Hsieh C L, Grange R, Pu Y and Psaltis D 2010 Biomaterials 312272

[5] Sandeau N, Le Xuan L, Chauvat D, Zhou C, Roch J-F and Brasselet S 2007 Opt. Express 1516051

[6] He Y, Zhang T, Zheng W, Wang R, Liu X, Xia Y and Zhao J 2010 Sensors Actuators B 14698

[7] Tachibana N, Yuasa M, Kida T, Yamazoe N and Shimanoe K 2011 Sens. Lett. 921

[8] Wang C, Yin L, Zhang L, Xiang D and Gao R 2010 Sensors 102088

[9] Feenstra J and Sodano H A 2008 J. Appl. Phys. 103124108

[10] Lu X, Zhang D, Zhao Q, Wang C, Zhang W and Wei Y 2006 Macromol. Rapid Commun. 2776

[11] Yuh J, Perez L, Sigmund W M and Nino J C 2007 Physica E 37254

[12] Li H, Wu H, Lin D and Panw W 2009 J. Am. Ceram. Soc. 922162

[13] Zhan S, Yu H, Li Y, Jiang B, Zhang X, Yan C and Ma S 2008 J. Disper. Sci. Technol. 291345

[14] Avinash B, Yiu-Wing M and Qian L 2011 Compos. Sci. Technol. 711435

[15] Rorvik P M, Grande T and Einarsrud M A 2011 Adv. Mater. 234007

[16] Sá P, Bdikin I, Almeida B, Rolo A and Isakov D 2012 Ferroelectrics $\mathbf{4 2 9} 48$

[17] Sahoo B and Panda PK 2012 Ceram. Int. 385189

[18] Emelyanov A Yu, Pertsev N A, Hoffmann-Eifert S, Bottger U and Waser R 2002 J. Electroceram. 95

[19] Zhao Z, Buscaglia V, Viviani M, Buscaglia M T, Mitoseriu L, Testino A, Nygren M, Johnsson M and Nanni P 2004 Phys. Rev. B 70024107

[20] Yen F-S, Hsiang H-I and Chang Y-H 1995 Japan. J. Appl. Phys. 346149

[21] Katta P, Alessandro M, Ramsier R D and Chase G G 2004 Nano Lett. 42215

[22] Bayan C, Levitt J M, Miller E, Kaplan D and Georgakoudi I 2009 J. Appl. Phys. 105102042

[23] Isakov D, Matos Gomes E, Belsley M, Almeida B, Martins A, Neves N and Reis R 2010 Europhys. Lett. 9128007

[24] Guinebretiere R 2007 X-ray Diffraction by Polycrystalline Materials (London: ISTE Ltd) Chapter 6

[25] Frey M H, Xu Z, Han P and Payne D 1998 Ferroelectrics 206337

[26] Stengel M and Spaldin N A 2006 Nature 443679

[27] Rabe K M, Ahn C H and Triscone J M 2007 Physics of Ferroelectrics: a Modern Perspective (Berlin: Springer)

[28] DiDomenico M, Wemple S H, Porto S P and Bauman R P 1968 Phys. Rev. 174522

[29] Venkateswaran U D, Naik V M and Naik R 1998 Phys. Rev. B 5814256

[30] Shiratori Y, Pithan S, Dornseier J and Waser R 2007 J. Raman Spectrosc. 381288

[31] Lines M E and Glass A M 1977 Principles and Applications of Ferroelectrics and Related Materials (Oxford: Clarendon)

[32] Vogt H 1974 Appl. Phys. 885

[33] Miller R C 1964 Phys. Rev. 134 A1313

[34] Pugachev A M, Kovalevskii V I, Surovtsev N V, Kojima S, Prosandeev S A, Raevski I P and Raevskaya S I 2012 Phys. Rev. Lett. 108247601 
[35] Isakov D, Volk T and Ivleva L 2009 Phys. Solid State 512334

[36] Denev S A, Lummen T T A, Barnes E, Kumar A and Gopalan V 2011 J. Am. Ceram. Soc. 942699

[37] Rodriguez E V, Araujo C B, Brito-Silva A M, Ivanenko V I and Lipovskii A A 2009 Chem. Phys. Lett. 467335

[38] Fox G R, Yamamoto J K, Miller D V, Cross L E and Kurtz S K 1990 Mater. Lett. 9284

[39] Kleemann W, Dec J, Shvartsman V, Kutnjak Z and Braun T 2006 Phys. Rev. Lett. 97065702
[40] Fiebig M, Fröhlich D, Lottermoser T and Maat M 2002 Phys. Rev. B 66144102

[41] Sun T, Wang X, Wang H, Zhang X, Cheng Z, Sun C Q and Liw L 2010 J. Am. Ceram. Soc. 932571

[42] Deng X, Wang X, Wen H, Kang A, Gui Z and Li L 2006 J. Am. Ceram. Soc. 891059

[43] Kalinin S and Gruverman A 2006 Scanning Probe Microscopy: Electrical and Electromechanical Phenomena at the Nanoscale (Berlin: Springer)

[44] Balke N, Bdikin I, Kalinin S and Kholkin A 2009 J. Am. Ceram. Soc. 921629 